PROCEEDINGS OF THE

AMERICAN MATHEMATICAL SOCIETY

Volume 140, Number 3, March 2012, Pages 987-998

S 0002-9939(2011)10994-8

Article electronically published on June 23, 2011

\title{
CONDITION NUMBER OF A SQUARE MATRIX WITH I.I.D. COLUMNS DRAWN FROM A CONVEX BODY
}

\author{
RADOSŁAW ADAMCZAK, OLIVIER GUÉDON, ALEXANDER E. LITVAK, ALAIN PAJOR, \\ AND NICOLE TOMCZAK-JAEGERMANN
}

(Communicated by Marius Junge)

\begin{abstract}
We study the smallest singular value of a square random matrix with i.i.d. columns drawn from an isotropic log-concave distribution. An important example is obtained by sampling vectors uniformly distributed in an isotropic convex body. We deduce that the condition number of such matrices is of the order of the size of the matrix and give an estimate on its tail behaviour.
\end{abstract}

\section{INTRODUCTION}

The quantitative behaviour of the smallest singular value of random matrices with i.i.d. random entries has attracted a lot of attention over the years. For Gaussian entries the problem has been investigated in [6] and [16, whereas for more general models of random matrices with i.i.d. entries, major results were recently obtained in [9, 13, 14, 17. In asymptotic geometric analysis one is interested in sampling vectors uniformly distributed over a convex body. In particular the entries of corresponding matrices are not necessarily independent. We develop this direction in the present paper, and we study an even more general case when the columns of random matrices are i.i.d. random vectors with an isotropic log-concave distribution. Our main result is a deviation inequality for the smallest singular value.

The first results concerning the smallest singular value of large random matrices were obtained in [6] and [16. The authors considered matrices with independent standard Gaussian entries and proved the following theorem (below $|\cdot|$ denotes the Euclidean norm on $\mathbb{R}^{n}$ ).

Received by the editors October 4, 2010 and, in revised form, December 6, 2010.

2010 Mathematics Subject Classification. Primary 52A23, 46B06, 60B20, 60E15; Secondary 52A20, 46B09.

Key words and phrases. Condition number, convex bodies, log-concave distributions, isotropic distributions, random matrix, norm of a random matrix, smallest singular number.

A part of this work was done when the first author held a postdoctoral position at the Department of Mathematical and Statistical Sciences, University of Alberta in Edmonton, Alberta. The position was sponsored by the Pacific Institute for the Mathematical Sciences. Research was partially supported by MNiSW Grant No. N N201 397437 and the Foundation for Polish Science.

The fifth author holds the Canada Research Chair in Geometric Analysis. 
Theorem 1.1 ([6, 16]). Let $n \geq 1$ and let $\Gamma$ be an $n \times n$ random matrix with i.i.d. $\mathcal{N}(0,1)$ entries. Then, for any $\varepsilon \geq 0$,

$$
\mathbb{P}\left(\inf _{x \in S^{n-1}}|\Gamma x| \leq \varepsilon n^{-1 / 2}\right) \leq C \varepsilon,
$$

where $C$ is an absolute constant.

Recently a lot of effort has been devoted to proving counterparts of the above theorem for matrices with general i.i.d. entries. Going in a geometrically motivated direction, we consider in this paper Ensembles (in the sense of random matrix theory) of matrices with i.i.d. columns distributed according to a log-concave isotropic probability measure. An important example of such an Ensemble is obtained by sampling vectors uniformly distributed in an isotropic convex body. The formal definition of this Ensemble is presented in Subsection 2.1 The main result of our paper is

Theorem 1.2. Let $n \geq 1$ and let $\Gamma$ be an $n \times n$ matrix with independent columns drawn from an isotropic log-concave probability $\mu$. For every $\varepsilon \in(0,1)$,

$$
\mathbb{P}\left(\inf _{x \in S^{n-1}}|\Gamma x| \leq c \varepsilon n^{-1 / 2}\right) \leq C \min \left\{n \varepsilon, \varepsilon+e^{-c \sqrt{n}}\right\} \leq C_{0} \varepsilon\left(\log \frac{2}{\varepsilon}\right)^{2},
$$

where $c, C$, and $C_{0}$ are absolute positive constants.

We would like to emphasize the difference between two terms under the minimum in formula (11). Contrary to the case e.g. of matrices with i.i.d. Bernoulli random entries, the left hand side of (11) tends to 0 as $\varepsilon \rightarrow 0$, due to the absolute continuity of $\log$-concave distributions. This is not the case for the second term $\varepsilon+\exp (-c \sqrt{n})$ under the minimum, but it has been achieved in the first term $\varepsilon n$ as well as in the right hand side of formula (11). In fact we conjecture that

$$
\mathbb{P}\left(\inf _{x \in S^{n-1}}|\Gamma x| \leq c \varepsilon n^{-1 / 2}\right) \leq C \varepsilon .
$$

In the setting of the log-concave Ensemble, the study of the limiting empirical distribution of the singular values has been done in [10. Weaker quantitative results in the direction of Theorem 1.2 were first obtained in [1. Similarly as in [9, 13, 14, the proof is based on the splitting of the sphere $S^{n-1}$ into two regions: the set of vectors whose norm is concentrated on a small number of coordinates and the set of other vectors. The main difficulty lies in the fact that we assume only independence of the column vectors of the matrix and not of all the entries. Therefore we develop several strategies to study different kinds of small ball probability estimates. One of the crucial new ingredients is Proposition 2.7 which will be essential in the setting of the log-concave Ensemble.

When combined with estimates for the operator norm of the matrix $\Gamma$, obtained recently in [2], the above theorem also yields a corollary about the tail behaviour of the so-called condition number of the matrix $\Gamma$ (denoted by $\kappa(\Gamma)$ ). The question about its behaviour for random matrices was raised by Smale 15] in connection with the stability of numerical algorithms for solving large systems of linear equations. Theorem 1.2 implies that for random matrices $\Gamma$ with independent log-concave isotropic columns, $\kappa(\Gamma) \leq C n$, similarly as for matrices with independent Gaussian entries $([6,16])$.

We also investigate the isotropic constant of isotropic log-concave measures (defined below in (5) ), a quantity of major importance in convex geometry. Although 
our estimates do not appear in the present proof of Theorem 1.2, they can be used for related problems; see [1. In particular Lemma 3.3 is a general result of a probabilistic nature and is of independent interest.

In Section 2, after presenting some preliminary facts (Subsection 2.2), we prove Theorem 1.2 (Subsection 2.3). We conclude the section with a tail inequality for the condition number of $\Gamma$ (Corollary 2.9). In Section 3 we present an estimate of the isotropic constant of the convolution of isotropic log-concave measures.

\section{Smallest Singular VAlue}

2.1. Basic definitions and notation. Throughout the paper $|\cdot|$ denotes the Euclidean norm and $\langle\cdot, \cdot\rangle$ the standard inner product on $\mathbb{R}^{n}$.

For an $n \times n$ matrix $\Gamma$, let $s_{1}(\Gamma) \geq s_{2}(\Gamma) \geq \ldots \geq s_{n}(\Gamma)$ be the singular values of $\Gamma$, i.e. the eigenvalues of the matrix $\sqrt{\Gamma \Gamma^{*}}$. In particular

$$
s_{1}(\Gamma)=\|\Gamma\|=\sup _{x \in S^{n-1}}|\Gamma x|,
$$

and if the matrix is invertible,

$$
s_{n}(\Gamma)=\frac{1}{\left\|\Gamma^{-1}\right\|}=\inf _{x \in S^{n-1}}|\Gamma x| .
$$

The condition number of a square matrix $\Gamma$ is defined as

$$
\kappa(\Gamma)=\|\Gamma\|\left\|\Gamma^{-1}\right\|=\frac{s_{1}(\Gamma)}{s_{n}(\Gamma)} .
$$

Let us now describe the model of random matrices we are interested in. Recall that a non-negative function $f: \mathbb{R}^{n} \rightarrow \mathbb{R}$ is called log-concave if for all $x, y \in \mathbb{R}^{n}$ and all $\theta \in(0,1), f((1-\theta) x+\theta y) \geq f(x)^{1-\theta} f(y)^{\theta}$. In this paper, a probability measure $\mu$ on $\mathbb{R}^{n}$ is said to be log-concave if it has density $f$, which is log-concave. It is called isotropic if it has mean zero and its covariance matrix is the identity, that is, for any $y \in \mathbb{R}^{n}$,

$$
\int_{\mathbb{R}^{n}}\langle x, y\rangle^{2} f(x) d x=|y|^{2} .
$$

A random vector $X$ in $\mathbb{R}^{n}$ is called log-concave (resp. isotropic) if its distribution is log-concave (resp. isotropic).

The log-concave Ensemble is defined to be the set of square $n \times n$ matrices $\Gamma$ whose columns $X_{1}, \ldots, X_{n}$ are independent copies of a log-concave isotropic random vector $X$ in $\mathbb{R}^{n}$.

2.2. Preliminary facts. In this section we collect some basic facts concerning general log-concave probability measures and random matrices, which will be used in the proof of Theorem 1.2 .

It is well known that log-concave vectors are stable under projections, which means that any orthogonal projection of an isotropic log-concave vector is an isotropic log-concave vector on the image of the projection. In particular, for any isotropic log-concave vector $X \in \mathbb{R}^{n}$ and any vector $u$ of Euclidean norm $1,\langle X, u\rangle$ is a $\log$-concave isotropic random variable on $\mathbb{R}$. Moreover, there exists an absolute constant $C$ such that any log-concave isotropic random variable on $\mathbb{R}$ has density bounded by $C$. 
It is also well known (4 4 ) that the convolution of log-concave functions is a logconcave function, which in probabilistic language means that the sum of log-concave vectors in $\mathbb{R}^{n}$ is a log-concave vector in $\mathbb{R}^{n}$.

We also recall some recent results concerning the behaviour of the Euclidean norm of an isotropic log-concave random vector. The following two inequalities, which yield a concentration in a shell, are two different results from [11] and [12] that we put together for convenience.

Theorem 2.1 (11, 12]). Let $N, n \geq 1$ be integers and let $X_{1}, \ldots, X_{N} \in \mathbb{R}^{n}$ be isotropic random vectors with log-concave densities. Then there exist absolute positive constants $c_{0}, C_{0}$ such that whenever $N \leq \exp (\sqrt{n})$, one has

$$
c_{0} \sqrt{n} \leq \min _{i \leq N}\left|X_{i}\right| \leq \max _{i \leq N}\left|X_{i}\right| \leq C_{0} \sqrt{n}
$$

with probability at least $1-\exp (-\sqrt{n})$.

Actually the above theorems were originally stated not for maxima and minima but just for a single log-concave vector. However the versions presented above follow easily by a union bound.

Since the proof of Theorem 1.2 involves approximation of arbitrary vectors in $S^{n-1}$ by vectors from $\varepsilon$-nets, we need to control the operator norm of a matrix $\Gamma$ from the log-concave Ensemble. To this end we will use the following result from [2] (it is an immediate consequence of Corollary 3.8 there).

Theorem 2.2. There exist positive constants $C_{1}$ and $c_{1}$ such that for any $n \geq 1$ and $K \geq 1$

$$
\mathbb{P}\left(\|\Gamma\| \geq C_{1} K \sqrt{n}\right) \leq \exp \left(-c_{1} K \sqrt{n}\right) .
$$

2.3. Proof of Theorem 1.2. Throughout this section $\Gamma$ denotes a matrix from the log-concave Ensemble (in fact Lemmas 2.3 and 2.5 hold for every random matrix).

With the notation of Theorem 1.2 we first prove the estimate

$$
\mathbb{P}\left(\inf _{x \in S^{n-1}}|\Gamma x| \leq c \varepsilon n^{-1 / 2}\right) \leq C \varepsilon+C \exp (-c \sqrt{n}) .
$$

The proof of this inequality relies on splitting the sphere $S^{n-1}$ into several regions (following [9, 13, 14], where an analogous construction was carried on in the case of matrices with independent entries). We use the following notation:

$$
\begin{aligned}
\text { Sparse } & =\operatorname{Sparse}(\delta)=\left\{x \in S^{n-1}:|\operatorname{supp}(x)| \leq \delta n\right\}, \\
\operatorname{Comp} & =\operatorname{Comp}(\delta, \rho)=\left\{x \in S^{n-1}: \operatorname{dist}(x, \operatorname{Sparse}(\delta)) \leq \rho\right\}, \\
\operatorname{Incomp} & =\operatorname{Incomp}(\delta, \rho)=S^{n-1} \backslash \operatorname{Comp}(\delta, \rho) .
\end{aligned}
$$

To control the behaviour of $|\Gamma x|$ for $x \in \operatorname{Incomp}(\delta, \rho)$, we will use the following lemma.

Lemma 2.3 (14). Let $X_{1}, X_{2}, \ldots, X_{n}$ denote the column vectors of $\Gamma$ and let $H_{k}$ denote the span of all column vectors, except the $k$-th column vector. Then for every $\rho, \delta \in(0,1)$ and every $\varepsilon>0$ one has

$$
\mathbb{P}\left(\inf _{x \in \operatorname{Incomp}(\delta, \rho)}|\Gamma x|<\varepsilon \rho n^{-1 / 2}\right) \leq \frac{1}{\delta n} \sum_{k=1}^{n} \mathbb{P}\left(\operatorname{dist}\left(X_{k}, H_{k}\right)<\varepsilon\right) .
$$


Proposition 2.4. For all $\rho, \delta, \varepsilon \in(0,1)$ we have

$$
\mathbb{P}\left(\inf _{x \in \operatorname{Incomp}(\delta, \rho)}|\Gamma x| \leq \rho \varepsilon n^{-1 / 2}\right) \leq C \frac{\varepsilon}{\delta},
$$

where $C$ is an absolute constant.

Proof. The column vectors of $\Gamma$ are the vectors $X_{1}, \ldots, X_{n}$. With probability 1 , they form a family of linearly independent vectors. For fixed $k$, let $H_{k}$ be the hyperplane spanned by $\left\{X_{i}: i \neq k\right\}$ and let $X_{k}^{*}$ be a vector of Euclidean norm 1 such that the space orthogonal to $H_{k}$ is generated by $X_{k}^{*}$. Then $X_{k}^{*}$ and $X_{k}$ are independent. Using Fubini's theorem, we obtain

$$
\mathbb{P}\left(\operatorname{dist}\left(X_{k}, H_{k}\right)<\varepsilon\right)=\mathbb{P}\left(\left|\left\langle X_{k}^{*}, X_{k}\right\rangle\right|<\varepsilon\right)=\mathbb{E}_{X_{k}^{*}} \mathbb{P}_{X_{k}}\left(\left|\left\langle X_{k}^{*}, X_{k}\right\rangle\right|<\varepsilon\right) .
$$

From the basic facts recalled in Subsection 2.2, for each fixed value of $X_{k}^{*},\left\langle X_{k}^{*}, X_{k}\right\rangle$ is a one-dimensional isotropic log-concave random variable; therefore its density $g$ is bounded by a universal constant $C$. This implies

$$
\mathbb{P}_{X_{k}}\left(\left|\left\langle X_{k}^{*}, X_{k}\right\rangle\right|<\varepsilon\right)=\int_{-\varepsilon}^{\varepsilon} g(t) d t \leq 2 C \varepsilon .
$$

Thus $\mathbb{P}\left(\operatorname{dist}\left(X_{k}, H_{k}\right)<\varepsilon\right) \leq 2 C \varepsilon$. By Lemma 2.3,

$$
\mathbb{P}\left(\inf _{x \in \operatorname{Incomp}(\delta, \rho)}|\Gamma x|<\varepsilon \rho n^{-1 / 2}\right) \leq \frac{1}{\delta n} \sum_{k=1}^{n} \mathbb{P}\left(\operatorname{dist}\left(X_{k}, H_{k}\right)<\varepsilon\right) \leq 2 C \frac{\varepsilon}{\delta}
$$

and the proof of (3) is completed.

The case of compressible vectors requires different tools. The following lemma is standard and restricts our study to the case of sparse vectors.

Lemma 2.5. Let $\rho, \delta \in(0,1)$ and $M \geq 1$. If

$$
\inf _{x \in \operatorname{Comp}(\delta, \rho / M)}|\Gamma x| \leq \rho \sqrt{n} \quad \text { and } \quad\|\Gamma\| \leq M \sqrt{n},
$$

then

$$
\inf _{y \in \operatorname{Sparse}(\delta)}|\Gamma y| \leq 2 \rho \sqrt{n} .
$$

Proof. Assume that there exists $x \in \operatorname{Comp}(\delta, \rho / M)$ such that $|\Gamma x| \leq \rho \sqrt{n}$ and $\|\Gamma\| \leq M \sqrt{n}$. Then by the definition of "compressible vectors", there exists $y \in$ Sparse $(\delta)$ such that $|x-y| \leq \rho / M$. Thus

$$
|\Gamma y| \leq|\Gamma x|+|\Gamma(y-x)| \leq \rho \sqrt{n}+\|\Gamma\| \rho / M \leq 2 \rho \sqrt{n} .
$$

To handle the case of sparse vectors, we will need the following version of Theorem 5.1 from $[3$ in the log-concave setting (applied with $N=n, m=\delta n, \theta=\sqrt{\delta} / 4$, $K=1)$.

Theorem 2.6. Let $n$ be a positive integer and let $\delta \in(0,1)$. Let $X_{1}, \ldots, X_{n} \in \mathbb{R}^{n}$ be independent random vectors with log-concave densities. Then

$$
\left.\sup _{x \in \operatorname{Sparse}(\delta)}|| \sum_{i=1}^{n} x_{i} X_{i}\right|^{2}-\sum_{i=1}^{n}\left|x_{i}\right|^{2}\left|X_{i}\right|^{2} \mid \leq C n \sqrt{\delta} \log \left(\frac{2}{\delta}\right)
$$


holds with probability larger than

$$
1-\exp \left(-\sqrt{\delta n} \log \left(\frac{2}{\delta}\right)\right)-\mathbb{P}\left(\max _{i \leq n}\left|X_{i}\right| \geq C_{0} \sqrt{n}\right),
$$

where $C$ is a positive universal constant and $C_{0}$ is the constant from Theorem 2.1.

Proposition 2.7. There exists a universal positive constant $c$ such that for any $\delta \in(0, c]$,

$$
\mathbb{P}\left(\inf _{x \in \operatorname{Sparse}(\delta)}|\Gamma x|<c \sqrt{n}\right) \leq 3 \exp (-\sqrt{\delta n}) .
$$

Proof. Applying Theorems 2.6 and 2.1, we obtain that

$$
\begin{aligned}
\inf _{x \in \text { Sparse }(\delta)}|\Gamma x|^{2} & \geq \inf _{x \in \text { Sparse }(\delta)} \sum_{i=1}^{n}\left|x_{i}\right|^{2}\left|X_{i}\right|^{2} \\
& -\left.\sup _{x \in \operatorname{Sparse}(\delta)}|| \sum_{i=1}^{n} x_{i} X_{i}\right|^{2}-\sum_{i=1}^{n}\left|x_{i}\right|^{2}\left|X_{i}\right|^{2} \mid \\
\geq & \left(c_{0}^{2}-C \sqrt{\delta} \log (2 / \delta)\right) n
\end{aligned}
$$

with probability at least

$$
\begin{aligned}
1-\exp (-\sqrt{\delta n} \log (2 / \delta))- & \mathbb{P}\left(\max _{i \leq n}\left|X_{i}\right| \geq C_{0} \sqrt{n}\right)-\mathbb{P}\left(\min _{i \leq n}\left|X_{i}\right| \leq c_{0} \sqrt{n}\right) \\
& \geq 1-3 \exp (-\sqrt{\delta n}) .
\end{aligned}
$$

This implies the result with appropriately chosen constant $c$.

Proposition 2.7 and Lemma 2.5 immediately yield the following statement, describing the case of compressible vectors.

Proposition 2.8. There exists an absolute constant $c_{2} \in(0,1)$ such that for any $M>1$ and $\delta \in\left(0, c_{2}\right]$

$$
\mathbb{P}\left(\inf _{x \in \operatorname{Comp}\left(\delta, c_{2} / M\right)}|\Gamma x| \leq c_{2} \sqrt{n} \&\|\Gamma\| \leq M \sqrt{n}\right) \leq 3 \exp (-\sqrt{\delta n}) .
$$

Proof of inequality (2). We apply Proposition 2.8 with $\delta=c_{2}$ and $M=C_{1}$, where $C_{1}$ is the constant from Theorem 2.2, to obtain

$$
\mathbb{P}\left(\inf _{x \in \operatorname{Comp}\left(c_{2}, c_{2} / C_{1}\right)}|\Gamma x| \leq c_{2} \sqrt{n}\|\Gamma\| \leq C_{1} \sqrt{n}\right) \leq 3 \exp \left(-\sqrt{c_{2} n}\right) .
$$

Thus, by Theorem 2.2 with $K=1$, we get for some positive universal constants $C$ and $c$,

$$
\mathbb{P}\left(\inf _{x \in \operatorname{Comp}\left(c_{2}, c_{2} / C_{1}\right)}|\Gamma x| \leq c_{2} \sqrt{n}\right) \leq 3 \exp \left(-\sqrt{c_{2} n}\right)+\exp \left(-c_{1} \sqrt{n}\right) \leq C e^{-c \sqrt{n}} .
$$

On the other hand, Proposition 2.4 with $\delta=c_{2}$ and $\rho=c_{2} / C_{1}$ gives for any $\varepsilon \in(0,1)$,

$$
\mathbb{P}\left(\inf _{x \in \operatorname{Incomp}\left(c_{2}, c_{2} / C_{1}\right)}|\Gamma x| \leq c_{2} C_{1}^{-1} \varepsilon n^{-1 / 2}\right) \leq \frac{C_{8}}{c_{2}} \varepsilon .
$$

Since $S^{n-1}=\operatorname{Incomp}\left(c_{2}, c_{2} / C_{1}\right) \cup \operatorname{Comp}\left(c_{2}, c_{2} / C_{1}\right)$ and $\varepsilon n^{-1 / 2} \leq n^{1 / 2}$, the above inequalities imply (2). 
To conclude the proof of Theorem 1.2 , it remains to show

$$
\mathbb{P}\left(\inf _{x \in S^{n-1}}|\Gamma x| \leq \varepsilon n^{-1 / 2}\right) \leq C n \varepsilon .
$$

Proof of inequality (4). Recall the notation from the proof of Lemma 2.3 and Proposition 2.4 namely that $H_{k}$ is the linear span of all the column vectors of $\Gamma$ except for $X_{k}$ and $X_{k}^{*}$ is a unit normal vector to $H_{k}$. It is elementary that

$$
\inf _{x \in S^{n-1}}|\Gamma x| \geq \min _{k \leq n} n^{-1 / 2} \operatorname{dist}\left(X_{k}, H_{k}\right)=\min _{k \leq n} n^{-1 / 2}\left|\left\langle X_{k}^{*}, X_{k}\right\rangle\right|
$$

(one simply uses the fact that any unit vector has at least one coordinate with absolute value not smaller than $n^{-1 / 2}$ ). Thus

$$
\begin{aligned}
\mathbb{P}\left(\inf _{x \in S^{n-1}}|\Gamma x|\right. & \left.\leq \varepsilon n^{-1 / 2}\right) \leq \mathbb{P}\left(\min _{k \leq n}\left|\left\langle X_{k}^{*}, X_{k}\right\rangle\right| \leq \varepsilon\right) \\
& \leq n \max _{k \leq n} \mathbb{P}\left(\left|\left\langle X_{k}^{*}, X_{k}\right\rangle\right| \leq \varepsilon\right) \leq C n \varepsilon,
\end{aligned}
$$

since (as was already mentioned in the proof of Proposition 2.4 $\left\langle X_{k}^{*}, X_{k}\right\rangle$ have densities bounded by a universal constant.

Finally we provide an estimate for the tail decay of the condition number of $\Gamma$.

Corollary 2.9. There are absolute positive constants $c$ and $C$ such that if $1 \leq t \leq \exp (c \sqrt{n})$, then

$$
\mathbb{P}(\kappa(\Gamma) \geq n t) \leq C \frac{1}{t}
$$

if $\exp (c \sqrt{n})<t \leq n \exp (c \sqrt{n})$, then

$$
\mathbb{P}(\kappa(\Gamma) \geq n t) \leq C \exp (-c \sqrt{n}) \leq C \frac{\log ^{2} t}{t} ;
$$

if $t>\exp (c \sqrt{n})$, then

$$
\mathbb{P}(\kappa(\Gamma) \geq n t) \leq C \frac{\sqrt{n} \log t}{t} \leq C \frac{\log ^{2} t}{t} .
$$

Proof. We are going to apply Theorems 1.2 and 2.2 Let $c, c_{1}, C$, and $C_{1}$ be constants from these theorems. Set $c_{3}=\min \left\{1, c, c_{1}\right\}$ and $C_{3}=\max \left\{1, C, C_{1}\right\}$. Note that for any $K \geq 1$

$$
\mathbb{P}(\kappa(\Gamma) \geq n t) \leq \mathbb{P}\left(\inf _{x \in S^{n-1}}|\Gamma x| \leq c_{3} \varepsilon n^{-1 / 2}\right)+\mathbb{P}\left(\|\Gamma\| \geq C_{3} K \sqrt{n}\right)
$$

where $\varepsilon=C_{3} K /\left(c_{3} t\right)$.

Now for $t \leq n \exp \left(c_{3} \sqrt{n}\right)$ we choose $K=1$, so by Theorems 1.2 and 2.2

$$
\mathbb{P}(\kappa(\Gamma) \geq n t) \leq C_{3}\left(\varepsilon+2 \exp \left(-c_{3} \sqrt{n}\right)\right) \leq \frac{C_{3}^{2}}{c_{3}} \frac{1}{t}+2 \exp \left(-c_{3} \sqrt{n}\right),
$$

which shows the first two cases.

For $t>\exp \left(c_{3} \sqrt{n}\right)$ we choose $K=\frac{\log t}{c_{3} \sqrt{n}}$, so by Theorems 1.2 and 2.2

$$
\mathbb{P}(\kappa(\Gamma) \geq n t) \leq C_{3}\left(\varepsilon n+\exp \left(-c_{3} K \sqrt{n}\right)\right)=C_{3} \frac{1}{t}\left(\frac{C_{3}}{c_{3}} \sqrt{n} \log t+1\right),
$$

which completes the proof. 


\section{ISOTROPIC CONSTANT OF A SUM OF I.I.D. RANDOM VECTORS IN $\mathbb{R}^{n}$}

Let $\mu$ be an isotropic log-concave probability measure on $\mathbb{R}^{n}$ with log-concave density $f$. We define the isotropic constant of the measure $\mu$ by

$$
L_{\mu}=f(0)^{1 / n} \text {. }
$$

For a log-concave isotropic random vector by $L_{X}$ we denote the isotropic constant of its distribution. Furthermore, if $\mu$ is an isotropic probability measure uniformly distributed on a convex body $K$, then $L_{\mu}$ is the so-called isotropic constant of $K$. The question of whether $L_{\mu}$ is bounded by a universal constant is one of the most important open problems of convex geometry.

The first version of Theorem 1.2 (as announced in [1]) involved the isotropic constant of column vectors of the matrix $\Gamma$. The argument was different than the one presented in this paper. Its proof required the control of the isotropic constant of the convolution of isotropic log-concave measures. Since a theorem providing such control is of independent interest, we present it now together with the proof.

Theorem 3.1. Let $X_{1}, \ldots, X_{n}$ be i.i.d. random vectors in $\mathbb{R}^{n}$ distributed according to a symmetric isotropic log-concave probability $\mu$ and let $x \in S^{n-1}$ and $Z=$ $x_{1} X_{1}+\ldots+x_{n} X_{n}$. Then $L_{Z} \leq C L_{\mu}$, where $C$ is a universal constant.

Note that in 1 1 we have deduced from this theorem another type of a small ball probability estimate than the one presented in Proposition 2.8 in the case of compressible vectors. Namely, we have proved the following proposition.

Proposition 3.2. Let $\Gamma$ be an $n \times n$ random matrix with independent columns $X_{1}, \ldots, X_{n}$ distributed according to a symmetric isotropic log-concave probability $\mu$. For every $M>1$ and $\delta, \rho \in(0,1)$ satisfying

$$
\rho \leq\left(\frac{C}{M^{\delta} L_{\mu}}\right)^{\frac{1}{1-\delta}}
$$

we have

$$
\mathbb{P}\left(\inf _{x \in \operatorname{Comp}(\delta, \rho /(2 M))}|\Gamma x| \leq \rho \sqrt{n} \&\|\Gamma\| \leq M \sqrt{n}\right) \leq e^{-c n}
$$

where $c$ and $C$ are positive absolute constants.

The proof of Theorem 3.1 is based on Lemma 3.3 below, which is a slightly modified version of a result of Gluskin and Milman [7, giving an $\ell_{2}$ lower bound for the norm defined on $\mathbb{R}^{n}$ by

$$
\left\|\left(\lambda_{1}, \ldots, \lambda_{n}\right)\right\|=\left(\int_{\mathbb{R}^{n}} \ldots \int_{\mathbb{R}^{n}}\left\|\sum_{i=1}^{m} \lambda_{i} x_{i}\right\|_{K}^{2} \prod_{i=1}^{m} f_{i}\left(x_{i}\right) d x_{i}\right)^{1 / 2},
$$

where $f_{1}, \ldots, f_{n}$ are probability densities on $\mathbb{R}^{n}$, and on a result of Junge (8]), which relates the isotropy constant of convolved log-concave probability measures with the norm $\|\cdot\|$.

Let $X_{1}, \ldots, X_{n}$ be independent isotropic log-concave symmetric random vectors in $\mathbb{R}^{n}$. Let $x \in S^{n-1}$ and set

$$
Z=x_{1} X_{1}+\ldots+x_{n} X_{n}
$$

Then it is well known (4]) that $Z$ is also an isotropic log-concave symmetric random vector in $\mathbb{R}^{n}$. 
Recall that $K$ is called a star body whenever $t K \subset K$ for all $0 \leq t \leq 1$, and in such a case $\|\cdot\|_{K}$ denotes its Minkowski functional, i.e. $\|x\|_{K}=\inf \{t>0: x \in t K\}$.

Lemma 3.3. Let $f_{1}, \ldots, f_{m}$ be densities of probability measures on $\mathbb{R}^{n}$ and let $K \subset \mathbb{R}^{n}$ be a star body containing the origin in its interior. Then for all $\lambda_{1}, \ldots, \lambda_{m}$ we have

$$
\int_{\mathbb{R}^{n}} \ldots \int_{\mathbb{R}^{n}}\left\|\sum_{i=1}^{m} \lambda_{i} x_{i}\right\|_{K}^{2} \prod_{i=1}^{m} f_{i}\left(x_{i}\right) d x_{i} \geq \frac{n}{n+2}|K|^{-2 / n} \sum_{i=1}^{m} \lambda_{i}^{2} r_{i}^{2},
$$

where $r_{i}^{2}=\int_{0}^{\infty}\left|\left\{x: f_{i}(x) \geq t\right\}\right|^{1+2 / n} d t \geq\left\|f_{i}\right\|_{\infty}^{-2 / n}$.

Proof. Let us recall that the symmetric decreasing rearrangement of a function $f: \mathbb{R}^{n} \rightarrow \mathbb{R}_{+}$is a function $f^{*}: \mathbb{R}^{n} \rightarrow \mathbb{R}$, which is equidistributed with $f$ (i.e. for all $\left.t \in \mathbb{R}_{+},\left|\left\{x: f^{*}(x) \geq t\right\}\right|=|\{x: f(x) \geq t\}|\right)$ and for $x, y \in \mathbb{R}^{n}$, if $|x| \leq|y|$, then $f^{*}(x) \geq f^{*}(y)$.

If $f$ is just the characteristic function of a set, then $f^{*}$ is the characteristic function of the ball of the same volume centered at the origin. In general one has the following "layer cake representation":

$$
f^{*}(x)=\int_{0}^{\infty}\left[\mathbf{1}_{\left\{y \in \mathbb{R}^{n}: f(y) \geq t\right\}}\right]^{*}(x) d t .
$$

Now let $D$ be the Euclidean ball of the same volume as $K$, centered at the origin. We will first prove that for all $t \in \mathbb{R}_{+}$one has

$$
\begin{aligned}
& \int_{\mathbb{R}^{n}} \ldots \int_{\mathbb{R}^{n}} \mathbf{1}_{\left\{\left(x_{i}\right)_{i=1}^{m}:\left\|\sum_{i=1}^{m} \lambda_{i} x_{i}\right\|_{K}^{2} \leq t\right\}} \prod_{i=1}^{m} f_{i}\left(x_{i}\right) d x_{1} \ldots d x_{m} \\
& \geq \int_{\mathbb{R}^{n}} \ldots \int_{\mathbb{R}^{n}} \mathbf{1}_{\left\{\left(x_{i}\right)_{i=1}^{m}:\left\|\sum_{i=1}^{m} \lambda_{i} x_{i}\right\|_{D}^{2} \leq t\right\}} \prod_{i=1}^{m} f_{i}^{*}\left(x_{i}\right) d x_{1} \ldots d x_{m} .
\end{aligned}
$$

It is a corollary from the Brascamb-Lieb-Luttinger inequality ([5]), which asserts that for any functions $g_{0}, \ldots, g_{m}: \mathbb{R}^{n} \rightarrow \mathbb{R}_{+}$and any $((m+1) \times k)$-matrix $\left(a_{i j}\right)$, we have

$$
\begin{aligned}
& \underbrace{\int_{\mathbb{R}^{n}} \ldots \int_{\mathbb{R}^{n}}}_{k} \prod_{i=0}^{m} g_{i}\left(\sum_{j=1}^{k} a_{i j} x_{j}\right) d x_{1} \ldots d x_{k} \\
\leq & \underbrace{\int_{\mathbb{R}^{n}} \ldots \int_{\mathbb{R}^{n}}}_{k} \prod_{i=0}^{k} g_{i}^{*}\left(\sum_{j=1}^{k} a_{i j} x_{j}\right) d x_{1} \ldots d x_{k} .
\end{aligned}
$$

Inequality (8) will follow if we substitute $g_{0}=\mathbf{1}_{\sqrt{t} K}$ and $g_{i}=f_{i}$ for $i \geq 1$ (notice that $\left.g_{0}^{*}=\mathbf{1}_{\sqrt{t} D}\right)$ with the appropriate choice of the matrix $\left(a_{i j}\right)$.

Since

$$
\|x\|_{K}^{2}=\int_{0}^{\infty} 2 t\left(1-\mathbf{1}_{\left\{\|x\|_{K} \leq t\right\}}\right) d t
$$


we conclude from (8) and from the symmetry of the $f_{i}^{*}$ that

$$
\begin{aligned}
\int_{\mathbb{R}^{n}} \ldots & \int_{\mathbb{R}^{n}}\left\|\sum_{i=1}^{m} \lambda_{i} x_{i}\right\|_{K}^{2} \prod_{i=1}^{m} f_{i}\left(x_{i}\right) d x_{i} \\
& \geq \int_{\mathbb{R}^{n}} \ldots \int_{\mathbb{R}^{n}}\left\|\sum_{i=1}^{m} \lambda_{i} x_{i}\right\|_{D}^{2} \prod_{i=1}^{m} f_{i}^{*}\left(x_{i}\right) d x_{i} \\
& =\sum_{i=1}^{m} \lambda_{i}^{2} \int_{\mathbb{R}^{n}} \ldots \int_{\mathbb{R}^{n}}\left\|x_{i}\right\|_{D}^{2} \prod_{i=1}^{m} f_{i}^{*}\left(x_{i}\right) d x_{i} .
\end{aligned}
$$

Since $|D|^{1 / n}=|K|^{1 / n}$, we have

$$
\|x\|_{D}=\left(\frac{\left|B_{2}^{n}\right|}{|K|}\right)^{1 / n}|x| .
$$

Now we can use (7) to get

$$
\begin{aligned}
\int_{\mathbb{R}^{n}}\|x\|_{D}^{2} f_{i}^{*}(x) d x & =\left(\frac{\left|B_{2}^{n}\right|}{|K|}\right)^{2 / n} \int_{\mathbb{R}^{n}}|x|^{2} f_{i}^{*}(x) d x \\
& =\left(\frac{\left|B_{2}^{n}\right|}{|K|}\right)^{2 / n} \int_{0}^{\infty} \int_{\mathbb{R}^{n}}|x|^{2}\left(\mathbf{1}_{\left\{y: f_{i}(y) \geq t\right\}}\right)^{*}(x) d x d t .
\end{aligned}
$$

The function $\left(\mathbf{1}_{\left\{y: f_{i}(y) \geq t\right\}}\right)^{*}$ is the indicator of the ball centered at the origin and having volume equal to $\left|\left\{y: f_{i}(y) \geq t\right\}\right|$. Therefore, integrating in polar coordinates,

$$
\begin{aligned}
\int_{\mathbb{R}^{n}}|x|^{2}\left(\mathbf{1}_{\left\{y: f_{i}(y) \geq t\right\}}\right)^{*}(x) d x & =\left(\frac{|\{y: f(y) \geq t\}|}{\left|B_{2}^{n}\right|}\right)^{1+2 / n} \int_{B_{2}^{n}}|x|_{2}^{2} d x \\
& =\frac{n}{n+2} \frac{|\{y: f(y) \geq t\}|^{1+2 / n}}{\left|B_{2}^{n}\right|^{2 / n}}
\end{aligned}
$$

Thus

$$
\int_{R^{n}}\|x\|_{D}^{2} f_{i}^{*}(x) d x \geq \frac{n}{n+2}|K|^{-2 / n} r_{i}^{2},
$$

where $r_{i}^{2}=\int_{0}^{\infty}\left|\left\{x: f_{i}(x) \geq t\right\}\right|^{1+2 / n} d t$. This concludes the proof of (6).

It remains to show that $r_{i}^{2} \geq\left\|f_{i}\right\|_{\infty}^{-2 / n}$. We know that

$$
\int_{0}^{\infty}\left|\left\{y: f_{i}(y) \geq t\right\}\right| d t=\int_{0}^{\left\|f_{i}\right\|_{\infty}}\left|\left\{y: f_{i}(y) \geq t\right\}\right| d t=1 .
$$

Hence by Hölder inequality,

$$
1 \leq\left(\int_{0}^{\left\|f_{i}\right\|_{\infty}}\left|\left\{y: f_{i}(y) \geq t\right\}\right|^{1+2 / n} d t\right)^{n /(n+2)}\left\|f_{i}\right\|_{\infty}^{2 /(n+2)} .
$$

Since $r_{i}^{2}=\int_{0}^{\infty}\left|\left\{x: f_{i}(x) \geq t\right\}\right|^{1+2 / n} d t=\int_{0}^{\left\|f_{i}\right\|_{\infty}}\left|\left\{x: f_{i}(x) \geq t\right\}\right|^{1+2 / n} d t$, we get the desired inequality.

Proof of Theorem 3.1. Let $f$ be the density of $\mu$ and let $g$ be the density of $Z$. By Lemma 2 in 8 , there exists a star-shaped body $K \subset \mathbb{R}^{n}$, with 0 in its interior such that

$$
g(0)^{1 / n}|K|^{1 / n}\left(\int_{\mathbb{R}^{n}}\|x\|_{K}^{2} g(x) d x\right)^{1 / 2} \leq C,
$$


for a certain universal constant $C$. On the other hand, by Lemma 3.3 we have

$$
\begin{aligned}
\left(\int_{\mathbb{R}^{n}}\|x\|_{K}^{2} g(x) d x\right)^{1 / 2} & =\left(\mathbb{E}\|Z\|_{K}^{2}\right)^{1 / 2}=\left(\mathbb{E}\left\|x_{1} X_{1}+\ldots+x_{n} X_{n}\right\|_{K}^{2}\right)^{1 / 2} \\
& \geq \frac{c}{|K|^{1 / n} f(0)^{1 / n}}\left(\sum_{i=1}^{n} x_{i}^{2}\right)^{1 / 2}=\frac{c}{|K|^{1 / n} f(0)^{1 / n}} .
\end{aligned}
$$

Putting these two inequalities together concludes the proof.

\section{ACKNOWLEDGMENT}

The work on this paper was completed when all the authors were attending the Thematic Program in Asymptotic Geometric Analysis in the Fields Institute in Toronto.

\section{REFERENCES}

[1] R. Adamczak, O. Guédon, A.E. Litvak, A. Pajor, N. Tomczak-Jaegermann, Smallest singular value of random matrices with independent columns, C. R. Math. Acad. Sci. Paris 346 (2008), no. 15-16, 853-856. MR2441920 (2009h:15009)

[2] R. Adamczak, A.E. Litvak, A. Pajor, N. Tomczak-Jaegermann, Quantitative estimates of the convergence of the empirical covariance matrix in log-concave Ensembles, J. Amer. Math. Soc. 23 (2010), no. 2, 535-561. MR2601042(2011c:60019)

[3] R. Adamczak, A. Litvak, A. Pajor, N. Tomczak-Jaegermann, Restricted isometry property of matrices with independent columns and neighborly polytopes by random sampling, Constructive Approximation, to appear (available online).

[4] C. Borell, Convex measures on locally convex spaces, Ark. Mat. 12 (1974), 239-252. MR0388475 (52:9311)

[5] H.J. Brascamp, E.H. Lieb, Best constants in Young's inequality, its converse, and its generalization to more than three functions, Adv. Math. 20 (1976), no. 2, 151-173. MR0412366 $(54: 492)$

[6] A. Edelman, Eigenvalues and condition numbers of random matrices, SIAM J. of Matrix Anal. and Applic. 9 (1988), pp. 543-560. MR964668 (89j:15039)

[7] E. Gluskin, V. Milman, Geometric probability and random cotype 2, GAFA, 123-138, Lecture Notes in Math., 1850, Springer, Berlin, 2004. MR2087156 (2005h:60031)

[8] M. Junge, Volume estimates for log-concave densities with application to iterated convolutions, Pacific J. Math. 169 (1995), 107-133. MR1346249(96i:46087)

[9] A. E. Litvak, A. Pajor, M. Rudelson, N. Tomczak-Jaegermann, Smallest singular value of random matrices and geometry of random polytopes, Adv. Math. 195 (2005), 491-523. MR2146352 (2006g:52009)

[10] A. Pajor, L. Pastur, On the limiting empirical measure of eigenvalues of the sum of rank one matrices with log-concave distribution, Studia Math. 195 (2009), no. 1, 11-29. MR 2539559 (2010h:15022)

[11] G. Paouris, Concentration of mass on convex bodies, Geom. Funct. Anal. 16, no. 5 (2006), 1021-1049. MR 2276533 (2007k:52009)

[12] G. Paouris, Small ball probability estimates for log-concave measures, Trans. Amer. Math. Soc. (to appear).

[13] M. Rudelson, Invertibility of random matrices: norm of the inverse, Annals of Math. 168, No. 2 (2008), 575-600. MR2434885 (2010f:46021)

[14] M. Rudelson, R. Vershynin, The Littlewood-Offord Problem and invertibility of random matrices, Adv. Math. 218, No. 2 (2008), 600-633. MR2407948 (2010g:60048)

[15] S. Smale, On the efficiency of algorithms of analysis, Bull. Amer. Math. Soc. 13 (1985), 87-121. MR799791 (86m:65061)

[16] S. Szarek, Condition numbers of random matrices, J. Complexity 7 (1991), no. 2, 131-149. MR.1108773 (92i:65086)

[17] T. Tao, V. Vu, Inverse Littlewood-Offord theorems and the condition number of random discrete matrices, Annals of Math. 169, No. 2 (2009), 595-632. MR2480613 (2010j:60110) 
Institute of Mathematics, University of Warsaw, Banacha 2, 02-097 Warszawa, POLAND

E-mail address: radamcz@mimuw.edu.pl

Université Paris-Est Marne-La-Vallée, Laboratoire d'Analyse et de Mathématiques Appliquées, 5, Boulevard Descartes, Champs sur Marne, 77454 Marne-la-Vallée, Cedex 2, France

E-mail address: olivier.guedon@univ-mlv.fr

Department of Mathematics and Statistical Sciences, University of Alberta, Edmonton, Alberta, Canada, T6G 2G1

E-mail address: alexandr@math.ualberta.ca

Université Paris-Est Marne-La-Vallée, Laboratoire d'Analyse et de Mathématiques Appliquées, 5, Boulevard Descartes, Champs sur Marne, 77454 Marne-la-Vallée, Cedex 2, France

E-mail address: Alain.Pajor@univ-mlv.fr

Department of Mathematics and Statistical Sciences, University of Alberta, Edmonton, Alberta, Canada, T6G 2G1

E-mail address: nicole.tomczak@ualberta.ca 